\title{
Thrombocytopenia resistent to standard therapy in lupus patients - analysis of 3 cases and clinical statement
}

\author{
Katarzyna Pawlak-Bus ${ }^{1,2}$, Piotr Leszczynski ${ }^{1,2}$, Mateusz Kokot ${ }^{2}$, Włodzimierz Samborski \\ ${ }^{1}$ Department of Rheumatology and Rehabilitation, Poznan University of Medical Sciences, Poland \\ ${ }^{2}$ Division of Rheumatology and Osteoporosis Jozef Strus Hospital, Poznan, Poland
}

Pawlak-Bus K, Leszczynski P, Kokot M, Samborski W. Thrombocytopenia resistent to standard therapy in lupus patients - analysis of 3 cases and clinical statement. Ann Agric Environ Med. 2018; 25(2): 229-233. doi: 10.26444/aaem/80708

\begin{abstract}
Hematologic disorders, including thrombocytopenia, are a common symptom of systemic lupus - Systemic Lupus Erythematosus (SLE). An important diagnostic and therapeutic issue is the severe and recurrent thrombocytopenia resistant to standard treatment. It requires extensive diagnostics and a multi-directional view on its causes beyond the autoimmune process. Currently, there is no single treatment regimen for these disorders. Corticosteroids (CS) are the first-line drugs, but their chronic use is a big problem, they are not always successful and often generate a number of complications, especially in moderate to high doses. In the case of thrombocytopenia associated with the activity of SLE, immunosuppressive therapy is the gold standard and may result in long-term remission of symptoms and clinical stabilization. The major problem is thrombocytopenia resulting from other causes, such as infection or medications. This study discusses severe recurrent thrombocytopenia on the basis of three clinical cases, analyzing both the various causes of disorders, and providing ways of management and treatment. Special attention is paid to the correlation of thrombocytopenia with the clinical and immunological activity of SLE. It seems that severe and refractory thrombocytopenia may be a critical point and largely determine the management and treatment possibilities of SLE.
\end{abstract}

Key words

thrombocytopenia, systemic lupus erythematosus (SLE), immunosuppressive treatment

\section{INTRODUCTION}

Hematologic disorders, including thrombocytopenia, are a common symptom of systemic lupus erythemtosus (SLE). The level of blood platelets (PLT) below $10 \mathrm{G} / 1$ is known as laboratory significant thrombocytopenia and is considered to be one of the criteria for the diagnosis of lupus according to the American College of Rheumatology (ACR) and others [1-3]. It can affect 7-30\% of SLE patients [4], and half of them suffer from thrombocytopenia at the beginning of diagnosis. Thrombocytopenia is also taken into account in the assessment of SLE activity by various international scales, such as SELENA-SLEDAI and BILAG $[5,6]$.

Increased peripheral platelet destruction related to the presence of antiplatelet antibodies is considered to be the most common cause of thrombocytopenia [7, 8]. Other reasons include impaired platelet production secondary to the presence of antibodies against thrombopoietin. The coexistence of SLE with the Anti-Phospholipid Antibody Syndrome (APS) or hemolytic anemia plays an important role in the etiology of hematological disorders [9]. Immunosuppressive drugs, such as azathioprine, or less frequently, hydroxychloroquine (HCQ), may also cause a reduction in the level of platelets. Very rare causes include thrombotic thrombocytopenic hemorrhagic diathesis, or thrombotic microangiopathic hemolytic anemia.

The course of thrombocytopenia can be very severe or even life-threatening. It has been proved that thrombocytopenia

Address for correspondence: Katarzyna Pawlak-Bus, Division of Rheumatology and Osteoporosis Jozef Strus Hospital, 61-285 Szwajcarska 3, Poznan, Poland e-mail:k.bus@makabu.net;

Received: 6 April 2016; accepted: 24 May 2017; first published on 21.11.2017 is an independent risk factor for increased mortality in SLE [10-12].

One of the major clinical and treatment problems is the profound and treatment-resistant thrombocytopenia in the course of SLE [8]. By default, the first line drugs include corticosteroids (CS), which give good results [10] in the treatment of acute thrombocytopenia. In contrast, chronic thrombocytopenia is less responsive to this treatment; therefore, it requires higher doses of CS and is more difficult to treat effectively. In the case of failure of standard corticosteroid treatment, one of the immunosuppressive drugs is normally used: azathioprine (AZA), mycophenolate mofetil (MMF), cyclosporine (CsA), or methotrexate (MTX) [13]. In certain cases, danazol treatment is also applied. If immunosuppressive therapy is ineffective, splenectomy is considered, although opinions on this procedure vary and are highly dependent on the individual clinical situation and expert opinion. The problem mainly concerns the possibility of performing the surgical treatment with low level of PLT, the effectiveness itself, and the risk of increased exposure to infections after the procedure $[14,15]$. In severe thrombocytopenia resistant to standard CS treatment, cyclophosphamide (CTX) is used intravenously in pulse therapy and immunoglobulins $[16$, 17]. As a parallel treatment, or in the case of CTX failure, plasmaphereses can be used as a method for removing the circulating antibodies that destroy platelets.

With the ineffectiveness of these methods, treatment with the use of rituximab (monoclonal anti-CD20 antibody, RTX) $[18,19]$ is considered, as well as autologic or allogenic transplantation of bone marrow stem cells $[20,21]$. The latest methods of treatment of thrombocytopenia include therapies using drugs that stimulate thrombopoiesis, 
i.e. thrombopoietin receptor agonists (romiplostim, eltrompobag) or a thrombopoietic growth factor (IL-11) [22-24]. However, to-date, no data is available on the use of these drugs in the treatment of thrombocytopenia in SLE patients.

Due to the immunological nature of hematological abnormalities in SLE, the use of platelet concentrates is not usually recommended. The exception is active bleeding or planned surgery, when the platelet level remains below $20 \mathrm{G} / \mathrm{l}$. Given that the majority of immunosuppressive drugs used to treat lupus can also cause or intensify thrombocytopenia, the decision about the choice of therapy can be sometimes very difficult. It requires medical experience in this subject and treatment individualization for each patient.

\section{CASE 1}

53-year-old male patient diagnosed with SLE was admitted to hospital because of extreme thrombocytopenia (level of PLT 0-1 G/L) and symptoms of hemorrhagic diathesis. In 2005 , the patient was diagnosed with SLE, based on typical skin lesions and arthritis. Thrombocytopenia (level of PLT 20-30 G/L) and antinuclear antibodies were also observed. In the beginning, the patient was been successfully treated with low dose of methylprednisolone (CS) $4 \mathrm{mg} / \mathrm{per}$ day and chloroquine (CQ) $250 \mathrm{mg} /$ per day. Afterwards, due to secondary ineffectiveness of the previously used standard therapy, the patient was started on biological therapy with epratuzumab anti-CD22 drug. For at least one year the patient was in a clinical trial (randomized and double blind, and therefore may have been receiving placebo). The patient was never unblinded.

On admission, the patient was found with extensive petechiae and bruising of the skin of the whole body, as well as on oral mucosa, inflammatory erythema on the face and torso. Laboratory tests (Tab. 1 and 2) showed undetectable levels of platelets (PLT - $0 \mathrm{G} / \mathrm{L}$ ). In addition, the deviations observed included accelerated erythrocyte sedimentation rate (ESR), the presence of anti-nuclear antibodies (ANA) with the presence of anti- RNP and anti-Sm antibodies, decreased levels of complement $\mathrm{C} 4$ and the presence of glycoprotein antibodies IIb/IIIa (GPIIb/IIIa). Despite the life-threatening condition of the patient, SLE activity on the SLEDAI scale was only rated 6 points (Tab. 3). Due to active bleeding, symptomatic treatment was carried out, together with transfusions of platelets concentrates, erythrocyte mass, followed by intravenous pulse therapy with high dose of CS (methylprednisolone at cumulative dose of 6.0g) added. In the absence of positive clinical effects, dexamethasone in the dose of $40 \mathrm{mg}$ i.v. was applied for 5 consecutive days. Unfortunately, previous treatment was not successful. Due to the persistent extreme thrombocytopenia (PLT 0-1 G/L), immunoglobulins were given intravenously in the dose of $2 \mathrm{~g} /$ per $\mathrm{kg}$ for 5 days. In total, the patient was treated with $140 \mathrm{~g}$ of IVIG. The critical decision was then made to add cyclophosphamid (CTX) as immunosuppressive therapy, which was initiated following pulse therapy (CTX 600mg i.v. every 2 weeks for a total of 6 doses). CS therapy was continued orally (prednisone at a dose of $0.5 \mathrm{mg} / \mathrm{kg}$ ). In the following days, platelet levels still remained at extremely low levels (PLT $0-1 \mathrm{G} / \mathrm{L}$ ), although there was no bleeding or new signs of hemorrhagic diathesis.
Table 1. Profile of immunological status in SLE patients

\begin{tabular}{|c|c|c|c|}
\hline & Case 1 & Case 2 & Case 3 \\
\hline $\begin{array}{l}\text { Positive antibodies } \\
\text { profile }\end{array}$ & $\begin{array}{c}\text { ANA }-137 \mathrm{U} / \mathrm{ml} \\
\text { anti-RNP } \\
\text { anti-Sm } \\
\text { anti-GPIIB/IIIA }\end{array}$ & $\begin{array}{c}\text { ANA - } 942 \mathrm{U} / \mathrm{ml} \\
\text { anti-RNP } \\
\text { anti-Sm } \\
\text { anti-dsDNA } \\
\text { anti-nucleosoms } \\
\text { anti-histones }\end{array}$ & $\begin{array}{c}\text { ANA - } 192 \mathrm{U} / \mathrm{ml} \\
\text { anti-dsDNA }\end{array}$ \\
\hline \multirow{2}{*}{$\begin{array}{l}\text { Immunological } \\
\text { activity of lupus }\end{array}$} & $\begin{array}{l}\text { anti-dsDNA } \\
\text { negative }\end{array}$ & $\begin{array}{c}\text { anti-dsDNA } \\
\uparrow 333.30\end{array}$ & $\begin{array}{c}\text { anti-dsDNA } \\
\uparrow 123.2\end{array}$ \\
\hline & $\begin{array}{c}\mathrm{C} 3 / \mathrm{C} 4 \\
\text { complement }-\downarrow\end{array}$ & $\begin{array}{c}\mathrm{C} 3 / \mathrm{C} 4 \\
\text { complement }-\downarrow\end{array}$ & $\begin{array}{c}\mathrm{C} 3 / \mathrm{C} 4 \\
\text { complement }-\downarrow\end{array}$ \\
\hline
\end{tabular}

ANA - antinuclear antibodies; anti-RNP - Anti-nuclear ribonucleoprotein antibodies, anti-Sm - anti Smith antibodies; anti-dsDNA - anti-double stranded DNA antibodies

Table 2. Standard laboratory results of described patients

\begin{tabular}{lccc}
\hline Tests & Case 1 & Case 2 & Case 3 \\
\hline Platelets $\left(10^{\%} / \mathrm{L}\right)$ & 0.0 & 17.0 & 2.0 \\
\hline WBC $\left(10^{9} / \mathrm{L}\right)$ & 4.4 & 2.8 & 20.6 \\
\hline RBC $\left(10^{9} / \mathrm{L}\right)$ & 3.5 & 2.5 & 2.7 \\
\hline $\mathrm{Hb}(\mathrm{g} / \mathrm{dl})$ & 10.6 & 8.0 & 8.8 \\
\hline Granulocytes $(\%)$ & 84.0 & 88.0 & 78.0 \\
\hline ESR $(\mathrm{mm} / \mathrm{h})$ & 63.0 & 20.0 & 42.0 \\
\hline CRP $(\mathrm{mg} / \mathrm{l})$ & 1.0 & 124.0 & 6.0 \\
\hline Creatinine $(\mathrm{umol} / \mathrm{l})$ & 64.0 & 261.0 & 98.0 \\
\hline ALT $(\mathrm{U} / \mathrm{l})$ & 50.0 & 64.0 & 10.0 \\
\hline AspAT $(\mathrm{U} / \mathrm{l})$ & 28.0 & 14.0 & 11.0 \\
\hline
\end{tabular}

WBC - white blood cells; RBC - red blood cells; $\mathrm{Hb}$ - haemoglobin; CRP - C-reactive protein; ALT - alanine aminotransferase; AST - aspartate aminotransferase

Table 3. Lupus activity according to SELENA-SLEDAI scale

\begin{tabular}{llccc}
\hline & & Case 1 & Case 2 & Case 3 \\
\hline SLEDAI score & before treatment & 6 & 51 & 10 \\
\hline SLEDAI score & after treatment & 4 & 26 & 8 \\
\hline
\end{tabular}

In the next phase of the scheduled treatment, the patient was subjected to a cycle of plasmapheresis. Three plasma exchange procedures were performed, $2,100 \mathrm{ml}$ of each plasma was exchanged with albumins and fresh frozen plasma concentrates, and immunoglobulins were then administered again $(2 \mathrm{~g} / \mathrm{kg}$ i.v) together with a second dose of CTX (600mg i.v). Only then a gradual increase in the level of platelets was observed. CS treatment was continued orally, gradually reducing the dose, and CTX therapy was performed under the planned scheme. In control, platelets remained at a satisfactory level (PLT 98-108-141 G/L) and no other signs of lupus activity were observed. CTX treatment was completed when the patient received $3,600 \mathrm{mg}$ of the drug in total. Then, to maintain remission, the therapy of mycophenolate mofetil (MMF) at dose of $2.0 \mathrm{~g} /$ day with good tolerance and efficacy, was included. In addition, antiCD22 (epratuzumab)/or placebo treatment in this patient was stopped, and not resumed. In subsequent controls, platelets and SLE activity continued to remained stable. 


\section{CASE 2}

A 19-year-old female patient was admitted to the Rheumatology Department ward in a very severe condition with a few weeks exacerbation of multiple organ symptoms of lupus, including profound thrombocytopenia. For several months, the patient had shown general weakness and leukopenia. On admission, the condition spread to the skin and mucous membranes. There were also observed hepatitis, acute pancreatitis, pleuritis, pericarditis, the presence of fluid in the abdominal cavity, as well as the characteristics of spread to the central nervous system of lupus nephritis and vasculitis. Laboratory tests revealed thrombocytopenia (PLT - 17 G/L), leukopenia, anemia, high levels of liver, kidney and pancreatic enzymes. Additionally, an increased rate of CRP (C-reactive protein) and the presence of high titers 1:640 of antinuclear antibodies (ANA) was detected. The profile of ANA consisted of antinative DNA, Sm, SSA, SSB and Ro52. Significant decreased levels were observed of C3 and C4 complement.

Given such a broad spectrum of symptoms, SLE activity was rated very high - 51 points on the SLEDAI scale. Initially, the patient required life-threatening procedures with symptomatic treatment. Erythrocyte mass transfusion and fresh frozen plasma were used, together with treatment with high-dose CS pulse therapy (3.0g of methylprednisolone i.v. in the cumulative dose for one cycle). A full dose of immunoglobulins was used (cumulative dose of $150 \mathrm{~g}$ i.v. for 5 consecutive days), and 4 series of plasmapheresis procedures with limited effectiveness. Due to active lupus and previously performed treatment there were also metabolic disorders, as well as neurological and psycho-organic complications. Additionally, progression of lupus nephritis with increased renal failure complicated with urinary tract infections was observed. Afterwards, intensive multi-directional systemic treatment was conducted, including classical antibacterial and antifungal treatment. Corticosteroids therapy was continued (prednisolone at a dose of $0.5 \mathrm{mg} / \mathrm{kg}$ orally), and a number of standard drugs used to improve the kidney and cardiovascular system functions.

Following a careful risk and benefits evaluation, including immunosuppressive therapy, it was decided to administer the patient with CTX with the dose adjusted to the parameters of renal function and thrombocytopenia. The performed scheduled was as follows: CTX i.v. 400mg every 2 weeks for a total of cumulative 6 doses. A gradual clinical improvement was observed during subsequent weeks. Renal function was improved, followed by decreased proteinuria, and the reduction of fluid in the body cavities. Moreover, a continuous increase in the level of blood platelets (PLT 43-148 G/L) and leukocytes was noted. SLE activity rated in SLEDAI significantly decreased from 51 to 26 points (Tab. 3). Already after the second pulse dose of CTX, the patient's condition allowed her discharge home. The CTX treatment was then continued under subsequent hospitalization according to the schedule. In the meantime, the dose of CS was gradually reduced. Further regression of clinical symptoms was observed, together with a definite improvement and stabilization of laboratory results. The level of platelets remained at $140 \mathrm{G} / \mathrm{L}$. After the end of the scheduled CTX therapy, the patient received a total cumulative dose of $3,200 \mathrm{mg}$, and MMF treatment was initialized at a dose of $3.0 \mathrm{~g} /$ day to maintain the long-term clinical and immunological remission of SLE.

\section{CASE 3}

A 41-year-old female patient was admitted due to extreme thrombocytopenia (PLT - $2 \mathrm{G} / \mathrm{L}$ ), with clinical symptoms of hemorrhagic diathesis. She had diagnosed with SLE 15 years ago on the basis of arthritis, skin lesions, nephropathy, and the presence of antinuclear antibodies. Throughout the period of the disease, the patient was chronically treated with high doses of CS with complications of this therapy. Additionally, she was treated with CQ as basic, and with modifying drugs such as CsA, RTX and MMF which were used step- bystep. The above treatment was not successful because of the deep and treatment-resistant thrombocytopenia which has been observed for two years. The patient, who noted life-saving indications, required multiple transfusions of platelet concentrates. On admission, the patient suffered from bleeding diathesis, livedo reticularis, swollen and tender joints and muscles, and signs and symptoms of iatrogenic hypercorticism. Laboratory results demonstrated thrombocytopenia (PLT - $2 \mathrm{G} / \mathrm{L}$ ), anemia, leukocytosis, increased ESR, elevated ANA, including positive anti-dsDNA and decreased C3 complement (Tab. 1, 2). SLE activity on the SLEDAI scale was rated at a moderate 10 points (Tab. 3). It was decided to use the standard CS treatment in pulse therapy (methylprednisolone 5x1,000 mg iv.) and CTX (according to the scheme: $400 \mathrm{mg}$ iv. every 2 weeks for a total of 6 cycles). Due to the resistance to CS and the previously used immunosupression, an immunoglobulin therapy was also included at a dose of $3 \mathrm{~g} / \mathrm{kg}$ (total dose of $240 \mathrm{~g}$ for 5 days). CS treatment was continued orally at a moderate dose (dexamethasone $2 \mathrm{mg}$ /day). Mild remission was achieved, but in spite of the immunosuppressive treatment, there were still recurring symptoms of hemorrhagic diatheses with low levels of platelets (PLT $-0-7 \mathrm{G} / \mathrm{L}$ ). Before administering the fourth cycle of CTX (so far, the patient has received a total of 1,200 mg CTX i.v.), abdominal pain, nausea and malaise were observed. Moreover, laboratory results revealed a significant increase in inflammatory parameters (ESR - $125 \mathrm{~mm} / \mathrm{h}$, CRP - $386 \mathrm{mg} / \mathrm{l}$ ). Further diagnostic tests, including imaging techniques, confirmed the presence of a perinephric abscess. Immunosuppressive therapy was abandoned, the patient required drainage of the abscess and intensive targeted antibiotic therapy. Long-term treatment resulted in a gradual stabilization of the patient's general condition, with clinical and laboratory improvement. Platelet levels significantly increased, reaching satisfactory values (PLT - from 34 to $100 \mathrm{G} / \mathrm{L}$ ). Oral treatment with low-dose of CS (dexamethasone $1 \mathrm{mg} /$ day) and CQ was continued with sustainable improvement.

\section{DISCUSSION}

Thrombocytopenia in the course of SLE is a common hematological disorder for which there is no specific treatment scheme [25]. Corticosteroids are the first-line therapy. Nevertheless, chronic corticosteroid treatment may lead to a well- known spectrum of complications. Taking into consideration various reasons for thrombocytopenia in SLE patients, the CS therapy is not always completely effective, as described in the above cases. Therefore, it should not be used in general as the only absolute standard. Normally used immunosuppressive drugs as additives to CS 
to treat active lupus with thrombocytopenia, such as MMF, CTX and CsA, can also generate a number of side-effects, e.g. granulocytopenia and severe infections, hemoglobin decrease, and paradoxically, a lower level of blood platelets. All things considered, the above treatment is extremely difficult and requires great clinical experience. In the absence of specific therapy, a severe problem in treatment-resistant thrombocytopenia is the approach towards the treatment of these hematological disorders, significant for the active and intricate lupus. Opinions and experiences in this regard vary and change often.

A new opportunity in SLE treatment is provided bybiological agents, such as rituximab (anti-CD20) [18]. It especially causes depletion of B lymphocytes and thus limits the overproduction of antibodies. Good results of this treatment are observed mainly in SLE with secondary antiphospholipid syndrome (APS) and catastrophic antiphospholipid syndrome (CAPS) with severe thrombocytopenia [26]. However, access to this therapy is still limited and off-label. Nevertheless, one of the above-described patients was probably targeted by epratuzumab (anti-CD22). Yet, nowadays, there is no additional data about its effect on platelets level. It may decrease SLE activity and therefore increase platelets level. On the contrary, it might cause and enhance thrombocytopenia, which was suspected in one of the described patients. It is essential to remember that the limitation of this observation is the unblinded situation of the patient in a clinical trial.

It should be emphasized how clinical manifestation was very different for the described patients and how varied the course of SLE was with accompanying thrombocytopenia, which can be seen in the SELENA-SLEDAI scoring scale (Tab. 3).

In the activity assessment by this scale, thrombocytopenia only scored one point, but it can be very severe and lifethreatening. Decreased blood platelet levels in serum may be the first symptom of SLE (case 1 and 3), it may be present for the entire duration of the disease or may appear during a severe exacerbation as an isolated, leading and treatmentresistant symptom.

Chronic thrombocytopenia is much more common and correlates less with disease activity (Cases 1 and 3), but is also more responsive to CS treatment. However, as described in the above cases, CS therapy was ineffective and potentially led to life-threatening side-effects.

Acute thrombocytopenia (Case 2) is less frequent in patients with SLE and is more resistant to treatment. It can occur as part of the manifested pancytopenia, and may accompany many other serious organ complications. Despite resistance to CS, it may undergo immunosuppressive treatment fairly quickly (Case 2). A correlation between the platelet deficit and activity of SLE in other systems allows for the classification of this symptom as part of the clinical manifestation of the active disease, and gives greater efficacy of the standard treatment. In the case of autoimmune disorders, aggressive immunosuppressive therapy can provide a good response, but not necessarily with immediate effects (Case 1). Treatment in such cases requires a lot of experience, prudence and consistent therapeutic strategy. However, we do not always have confident knowledge of the causes of thrombocytopenia, even after thorough analysis of all its possible causes (Cases 1 and 3). The etiology of hematological disorders cannot exclude the effect of drugs, infections and various unknown factors that may induce or intensify thrombocytopenia in the course of SLE. Given that the majority of immunosuppressive drugs used to treat lupus can also cause or intensify thrombocytopenia, the decision about the choice of therapy can be very difficult. In patients chronically treated with CS and in whom the risk of infections and their complications is relatively high [27], thrombocytopenia may be one of the symptoms of bacterial inflammation, such as an abscess (Case 3).

In the cases cited, all known methods of therapy were used (Fig. 1). This illustrates the importance and scale of the problem. Hence, more specific treatments of hematological disorders secondary to SLE are continuously being explored. Recent reports show that HCQ used in patients with longterm immune thrombocytopenia with documented failure of various other standard methods of treatment, results in a very good effect in up to $83 \%$ of cases. This treatment primarily allows reduction of CS doses in most patients [13], and sometimes even discontinuation of CS treatment. A beneficial and permanent effect of increased blood platelets at more than $100 \mathrm{G} / \mathrm{L}$ was obtained by applying a long-term HCQ regimen over 5 months. The patients presented above (Cases 1-3) were also treated with an anti-malarial drug, but not all of them had the chance of obtaining the effects of this therapy due to its duration. Considering the fact that HCQ is currently the basic therapy in SLE, the effect of future treatments of thrombocytopenia should be much improved.

Thrombocytopenia is not only one of the criteria for the diagnosis or classification of SLE, but above all, it is a critical point in the treatment of systemic lupus. Hematologic disorders associated with SLE can be both a symptom of disease activity and a symptom of instability due to another cause. Because of the complex etiology of thrombocytopenia, careful analysis of its causes appears to be mandatory in every case. Recurrent severe thrombocytopenia in active SLE requires immunosuppressive treatment. Both cyclophosphamide and mycophenolate mofetil may be effective. Treatments that cause depletion of B lymphocytes (rituximab or epratuzumab) or the newly available drug belimumab, which inactivates $\mathrm{B}$ cells, are very promising and give encouraging results. Yet, this therapeutic regimen ought to be further investigated and proved in future clinical studies and daily practice.

\section{REFERENCES}

1. Tan EM, Cohen AS, Fries JF, et al. The 1982 revised criteria for the classification of systemic lupus erythematosus. Arthritis Rheum. 1982; 25(11): 1271-1277.

2. Petri M, Orbai AM, Alarco'n GS et al. Derivation and Validation of the Systemic Lupus International Collaborating Clinics Classification Criteria for Systemic Lupus Erythematosus. Arthritis Rheum. 2012; 64(8): 2677-2268.

3. Hochberg MC. Updating the American College of Rheumatology revised criteria the classification of systemic lupus erythematosus. Arthritis Rheum. 1997; 40(9): 1725.

4. Hepburn AL, Narat S, Mason J. The management of peripheral blood cytopenias in systemic lupus erythematosus. Oxford Rheumatol J. 2010; 49: 2243-2254.

5. Bombardier C, Gladman DD, Urowitz MB, et al. Derivation of the SLEDAI. A disease activity index for lupus patients. The Committee on Prognosis Studies in SLE. Arthritis Rheum. 1992; 35: 630-640.

6. Hay EM, Bacon PA, Gordon C, et al. The BILAG index: a reliable and valid instrument for measuring clinical disease activity in systemic lupus erythematosus. Q J Med. 1993; 86: 447-458. 
7. Michel M, Lee K, Piette JC, et al. Platelet autoantibodies and lupusassociated thrombocytopenia. Br J Heamatol. 2002; 119: 354-358.

8. Nossent JC, Swaak AJ. Prevalence and significance of haematological abnormalities in patients with systemic lupus erythematosus. Q J Med. 1990; 74: 319-330.

9. Lipp E, von Felten A, Sax H, et al. Antibodies against platelet glycoproteins and antiphospholipid antibodies in autoimmune thrombocytopenia. Eur J Haematol. 1998; 60: 283-288.

10. Mok CC, Lee KW, Ho CT, et al. A prospective study of survival and prognostic indicators of systemic lupus erythematosus in a southern Chinese population. Rheumatology 2000; 39: 399-406.

11. Cervera R, Kamashta MA, Font J, et al. Morbidity and mortality is systemic lupus erythematosus during a 5-year period. A multicenter prospective study of 1000 patients. European Working Party on Systemic Lupus Erythematosus, Medicine 1999; 78: 167-175.

12. Khellaf M, Chabrol A, Mahevas M, et al. Hydroxychloroquine is a good second-line treatment for adults with immune thrombocytopenia and positive antinuclear antibodies. Am J Hematol. 2013; 89(2): 194-198.

13. Leszczyński P, Pawlak-Bus K. New treatment strategy including biological agents in patients with systemic lupus erythematosus. Pol Arch Med Wewn. 2013; 123(9): 482-490.

14. Jacobs P, Wood L, Dent DM. Splenectomy and the thrombocytopenia of systemic lupus erythematosus. Ann Intern Med. 1986; 105: 971-972.

15. Gruenberg JC, VanSlyck EJ, Abraham JP. Splenectomy in systemic lupus erythematosus. Am J Surg. 1988; 155: 391-394.

16. Hall S, McCormick JL Jr, Greipp PR, et al. Splenectomy does not cure the thrombocytopenia of systemic lupus erythematosus. Ann Intern Med. 1985; 102: 325-328.

17. Boumpas DT, Barez S, Klippel JH, et al. Intermittent cyclophosphamide for the treatment of autoimmune thrombocytopenia in systemic lupus erythematosus. Ann Intern Med. 1990; 112: 674-677.
18. Maier WP, Gordon DS, Howard RF, et al. Intravenous immunoglobulin therapy in systemic lupus erythematosus - associated thrombocytopenia. Arthritis Rheum. 1990; 33: 1233-1239.

19. Lehembre S, Macario-Barrel A, et al. Rituximab treatment for immune thrombocytopenia associated with systemic lupus erythematosus. Ann Dermatol Venereol. 2006; 133: 53-55.

20. Ramos-Casals M, Brito-Zeron P, Munoz S, et al. A systematic review of the off-label use of biological therapies in systemic autoimmune diseases. Medicine. 2008; 87: 345-364.

21. Traynor AE, Barr WG, Rosa RM, et al. Hematopoietic stem cell transplantation for severe and refractory lupus. Analysis after five years and fifteen patients. Arthritis Rheum. 2002; 46: 2917-2923.

22. Lisukova IA, Sizikova SA, Kulagin AD, et al. High-dose immunosuppression with autologous stem cell transplantation in severe refractory systemic lupus erythematosus. Lupus 2004; 13: 89-94.

23. Bussel JB, Cheng G, Saelh MN, et al. Eltrombobag for treatment of chronic idiopatic thrombocytopenia purpura. N Engl J Med. 2007; 357: 2237-2247.

24. Bussel JB, Kuter DJ, Pullarkat V, et al. Safety and efficacy of long-term treatment with romiplostim in thrombocytopenic patients with chronic ITP. Blood. 2009; 113: 2161-2171.

25. Feinglass S, Deodhar A. Treatment of lupus- induced thrombocytopenia with recombinant human interleukin-11. Arthritis Rheum. 2001; 44: $170-175$.

26. Bashal F. Hematological Disorders in Patients with Systemic Lupus Erythematosus Open Rheumatol J. 2013; 7: 87-95.

27. Kumar D, Robert A, Roubey S. Use of Rituximab in the Antiphospholipid Syndrome. Curr Rheumatol Rep. 2010; 12: 40-44.

28. Ruiz-Irastorza G, Olivares N, Ruiz-Arruzal I, et al. Predictors of major infections in systemic lupus erythematosus. Arthritis Res Ther. 2009; 11(4): R109. 